\title{
Effects of a New Human Recombinant MnSOD in the Treatment of Photoaging and Actinic Keratosis
}

\author{
Maria Grazia Ruggiero ${ }^{1}$, Maria Laura Pollio ${ }^{1}$, Antonietta Schiattarella ${ }^{2}$, Antonella Borrelli ${ }^{2}$, \\ Aldo Mancini ${ }^{2}$, Alessandra Pica ${ }^{1 *}$, Patrizia Forgione ${ }^{3}$ \\ ${ }^{1}$ Department of Biology, University of Naples Federico II, Naples, Italy; ${ }^{2}$ National Cancer Institute “G. Pascale”, Naples, Italy; ${ }^{3}$ Unit \\ of Dermatology, “Ascalesi” Hospital, Naples, Italy. \\ Email: *alessandra.pica@unina.it
}

Received May 23 ${ }^{\text {rd }}, 2013$; revised June 22 ${ }^{\text {nd }}, 2013$; accepted June $30^{\text {th }}, 2013$

Copyright (c) 2013 Maria Grazia Ruggiero et al. This is an open access article distributed under the Creative Commons Attribution License, which permits unrestricted use, distribution, and reproduction in any medium, provided the original work is properly cited.

\begin{abstract}
Physiological processes, as aerobic metabolism and inflammatory response, generate reactive oxygen species (ROS) that may induce cellular injury when their amount is increased and antioxidant defense mechanisms are overwhelmed. Also, ROS are generated following UV skin irradiation able to deplete the natural antioxidant defenses in the skin. The increase in exposure to UV may lead to photoaging and precancerous skin lesions (actinic keratosis). New antioxidant strategies in the prevention and therapy of skin lesions are urgently needed. In this study, we evaluated the antioxidant efficacy of a recombinant form of human manganese superoxide dismutase able to inhibit reactive oxygen species production in some patients affected by severe photoaging and actinic keratosis.
\end{abstract}

Keywords: Photoaging; Actinic Keratosis; MnSOD; Skin Cancer Lesions

\section{Introduction}

The skin is one of the largest organs of the human body, whose main function is to maintain its own integrity, and acts as a protective barrier against external agents, noxious substances and pathogens [1]. Some exogenous factors, in particular the UV rays, can modify the functionality of the skin barrier. UV can induce both quantitative and qualitative changes of immunocompetent cells, and may alter the immune surveillance to skin cancers. The ultraviolet irradiation stimulates the secretion of cytokines and growth factors by keratinocytes that intervene on immunocompetent cells [2]. UVB (290 - $320 \mathrm{~nm})$ and UVA (320 - $400 \mathrm{~nm}$ ) penetrate and pass through the epidermis, reaching the underlying dermis. UVB and UVA interacting with endogenous chromophores and photosensitizers, generate the production of reactive oxygen species (ROS) [3]. The reactive oxidants of ROS include superoxide anion $\left(\mathrm{O}_{2}^{-}\right)$, singlet oxygen $\left(\mathrm{O}_{2}\right)$, hydrogen peroxide $\left(\mathrm{H}_{2} \mathrm{O}_{2}\right)$, and the hydroxyl radical $(\mathrm{OH})$. In according to Masaki [4], ROS, generated by endogenous or exogenous processes, induce skin erythema and have an established role in UV-induced skin aging, characterized by wrinkles. The skin has an enzyme system which mi-

\footnotetext{
"Corresponding author.
}

nimizes the damaging effect of ROS and helps to maintain the homeostatic balance, such as glutathione reductase, catalase, and superoxide dismutase. The superoxide dismutases (SODs) are enzymes of remarkable pharmacological interest for their potential role in the prevention of all the pathologies caused by oxidative damage [5]. SODs are involved in antioxidant defense in almost all cells, since they catalyze the dismutation of superoxide radical into hydrogen peroxide, which is subsequently converted to water and oxygen by the enzyme catalase [6]. Many studies have shown that antioxidant activity, mainly the one exerted by manganese superoxide dismutase (MnSOD), is reduced in various skin cancers [7]. This paper focuses on a new form of SOD, extracted from human liposarcoma cells, which showed peculiar characteristics both in vivo and in vitro. It indeed exhibits a selective cytotoxic power for cells that over-expressing estrogen receptor sites. This isoform was called LSA-type MnSOD. This protein, has the same enzymatic activity common to all SODs, but differs structurally and functionally from its corresponding native. First, it is secreted by LSA cells in the medium, whereas the native MnSOD is synthesized and resides in the mitochondrial matrix [8]. In addition, the recombinant form of LSA-MnSOD (rMnSOD) not only exerts the same radioprotective ef- 
fect on normal cells and organisms as any other MnSOD, but it also exerts a radiosensitizing action on tumor cells. It was also demonstrated that healthy mice, exposed to lethal doses of ionizing radiation and to daily injections of rMnSOD, were protected from radiation damage and were still alive 30 days after the $\mathrm{X}$ ray, while the controls injected only with PBS solution, died 7 - 8 days later [5]. The remarkable ability of rMnSOD to neutralize free radicals, that occur in damaged tissues, has also been demonstrated by the complete recovery from a severe necrosis of the head, neck, and fins of a specimen of loggerhead sea turtle Caretta caretta. The daily topical treatment of Caretta caretta lesions with rMnSOD over a month, enzymatically transformed all the free radicals present in the lesion thus completely, avoiding necrosis and tissue breakdown, leading to the generation of new tissue [9].

The oil in water emulsion based on rMnSOD was tested on patients who had given their informed consent. The emulsion was applied twice daily on the skin lesions, for 60 days. Patients enrolled in this study belonged to skintypes I and II, (Fritzpatrick Skin-Types), and had severe photoaging. In two cases, advanced actinic keratosis too was present.

\subsection{Case 1}

P. F., a 55-year-old woman, showed severe skin elastosis with superficial and deep wrinkles, and solar lentigo (Figure 1(A)).

She applied the rMnSOD emulsion every morning and evening for 60 days.

At the end of the clinical observational study, there was clear evidence of a substantial improvement of the skin elastosis and wrinkles depth, and also a reduction in size and number of solar lentigo (Figure 1(B)).

\subsection{Case 2}

A. N., a 78-year-old man with skin-type I, with severe photoaging, extensive skin teleangectasia, and actinic keratosis localized in the right malar region, some of which appear as very advanced lesions (Figure 2(A)).

He had already been subjected to photodynamic therapy for 6 months, and had undergone surgical removal of two epitheliomas in the fronto-parietal area.

The schedule of topical treatment based on rMnSOD, as for the previous patient, was 60 days every morning and evening.

At the end of the follow-up study, he showed clinical subtotal regression of all actinic keratosis, color improvement of skin and wrinkles depth (Figure 2(B)).

\subsection{Case 3}

C. R., an 83-year-old man, was affected by multiple epi-
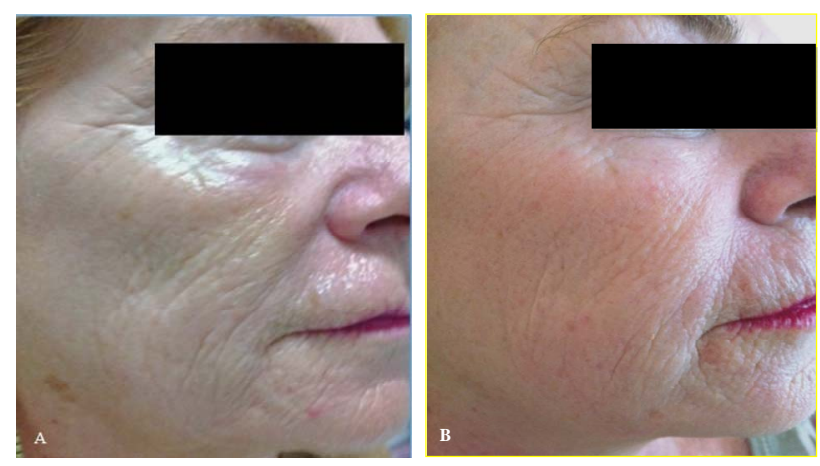

Figure 1. (A) At time 0, patient with severe solar elastosis and solar lentigo; (B) At 2 months, marked elastosis improvement and solar lentigo regression.
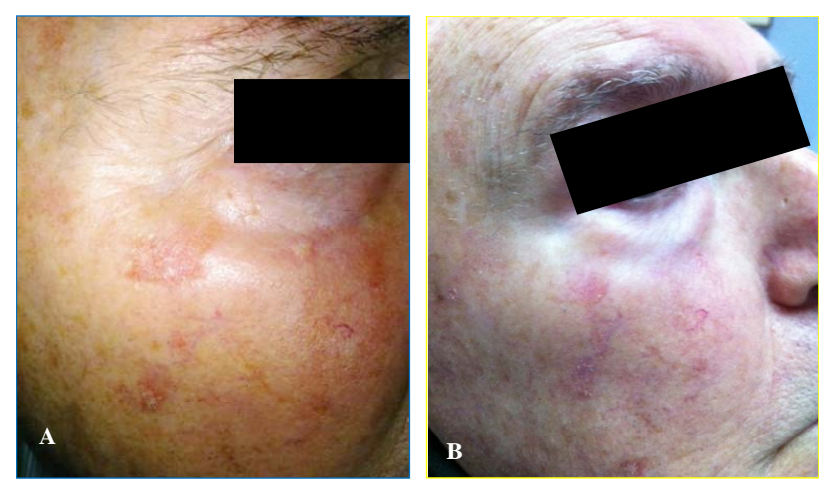

Figure 2. (A) At time 0, patient with advanced actinic keratosis and photoaging; (B) At 2 months, total regression of actinic keratosis of the bottom eyelid's margin, subtotal reduction of cheek kearatosis.

thelioma, actinic keratosis and severe photoaging over all his skin. In particular legs showed thin and erythematous skin with the presence of severe dyskeratosis and clearly neoplastic areas (Figure 3(A)). In this case the protocol required surgical therapy for the two facial epitheliomas, and photodynamic therapy for the actinic keratosis in frontal and malar region.

Regarding the lower limbs, a topical application of rMnSOD was employed twice daily for 60 days.

At the end of the 2-month follow-up study, there was clear evidence of a subtotal regression of precancerous lesions, and of a recovered epidermis, showing an increased firmness of the skin and improving of the skin color (Figure 3(B)).

\subsection{Case 4}

M.B., a 78-year-old woman, with skin-type I, photo damaged skin and widespread presence of solar lentigo (Figure 4(A)).

After two months of treatment with topical rMnSOD, we observed a subtotal regression of solar lentigo, skin firmness increase and improvement in skin brightness (Figure 4(B)). 

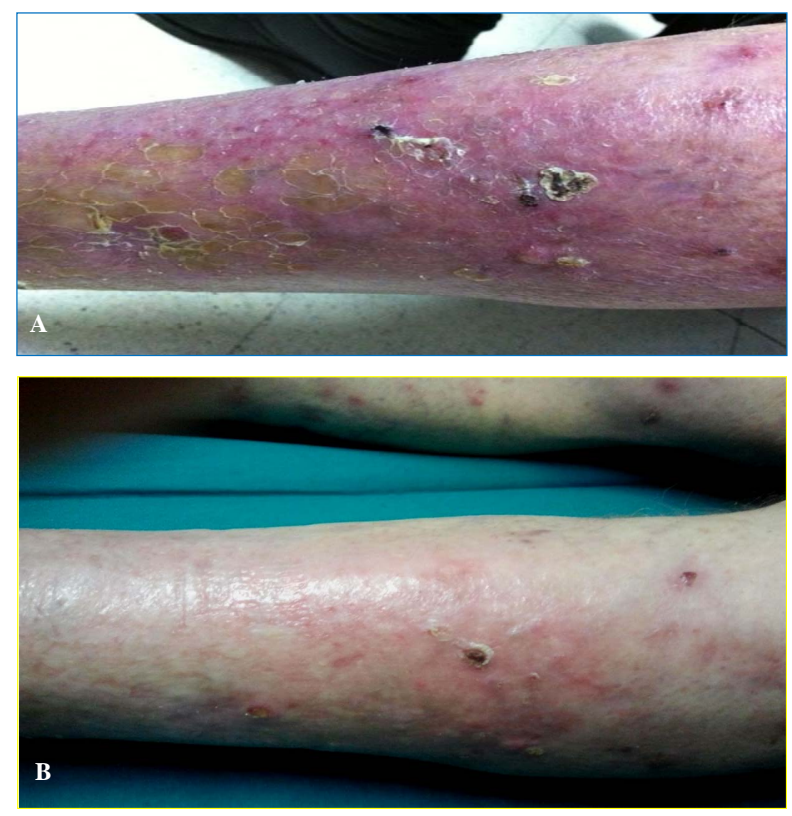

Figure 3. (A) Time 0, photodamaged skin severe and advanced actinic keratosis; (B) At 2 months, subtotal regression of precancerous lesions and increased firmness of the skin and improving of the skin color.
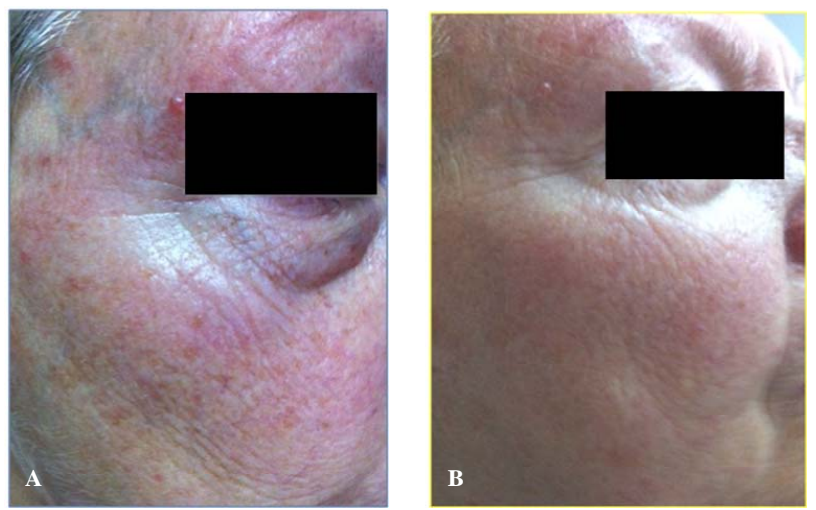

Figure 4. (A) Time 0, photodamaged skin with widespread solar lentigo; (B) at 2 months, subtotal regression of solar lentigo, skin firmness increase and significant improvement in the brightness of the skin.

\section{Discussion}

ROS generation represents one of the main factor causing skin alterations since they induce changes in gene expression determining collagen degradation and elastin accumulation. As a result of the present study, it can be affirmed that rMnSOD is a very interesting molecule due to its marked anti-inflammatory and reparative power. Furthermore, as previously demonstrated [10], its ability to modulate p53 brings about a clear improvement of precancerous or neoplastic lesions.

Overall, rMnSOD offers interesting, new perspectives for the treatment of chronoaging and photoaging. Due to the few cases so far analyzed, to date, the main role of to- pical treatment with rMnSOD has been observed in supporting of photodynamic therapy and in the prevention and in the supportive treatment of actinic keratosis.

\section{REFERENCES}

[1] C.-H. Lee, S.-B. Wu, C.-H. Hong, H.-S. Yu and Y.-H. Wei, "Molecular Mechanism of UV-Induced Apoptosis and Its Effects on Skin Residential Cells: The Implication in UV-Based Phototherapy," International Journal of Molecular Sciences, Vol. 14, No. 3, 2013, pp. 6414-6435. doi:10.3390/ijms14036414

[2] P. Santoianni and M. Nino, " Sunlight and Cancer Risk,” Italian Journal of Dermatology and Venereology, Luce Solare e Rischio Oncologico, Giornale Italiano di Dermatologia e Venereologia, Vol. 138. No. 6, 2003, pp. 455-464.

[3] F. Debacq-Chainiaux, C. Leduc, A. Verbeke and O. Toussaint, "UV, Stress and Aging," Dermato-Endocrinology, Vol. 4, No. 3, 2012, pp. 236-240. doi:10.4161/derm.23652

[4] H. Masaki, "Role of Antioxidants in the Skin: Anti-Aging Effects," Journal of Dermatological Science, Vol. 58, No. 2, 2010, pp. 85-90. doi:10.1016/j.jdermsci.2010.03.003

[5] A. Borrelli, A. Schiattarella, R. Mancini, B. Morrica, V. Cerciello, M. Mormile, V. D’Alesio, L. Bottalico, F. Morelli, M. D’Armiento, F. P. D’Armiento and A. Mancini, "A Recombinant MnSOD Is Radioprotective for Normal Cells and Radiosensitizing for Tumor Cells," Free Radical Biology and Medicine, Vol. 46, No. 1, 2009, pp. 110116. doi:10.1016/j.freeradbiomed.2008.10.030

[6] M. W. Epperly, J. E. Gretton, C. A Sikora, M. Jefferson, M. Bernarding, S. Nie and J. S. Greenberger, "Mitochondrial Localization of Superoxide Dismutase Is Required for Decreasing Radiation-Induced Cellular Damage,” Radiation Research, Vol. 160, No. 5, 2003, pp. 568-578. doi:10.1667/RR3081

[7] D. Robbins and Z. Yunfeng, "The Role of Manganese Superoxide Dismutase in Skin Cancer,” Enzyme Research, Vol. 2011, No. 2011, 2011, Article ID: 409295. doi:10.4061/2011/409295

[8] A. Mancini, A. Borrelli, A. Schiattarella, S. Fasano, A. Occhiello, A. Pica, P. Sher, M. Tommasino, J. P. F. Nüesch and J. Rommelaere, "Tumor Suppressive Activity of a Variant Isoform of Manganese Superoxide Dismutase Released by a Human Liposarcoma Cell Line,” International Journal of Cancer, Vol. 119, No. 4, 2006, pp. 932-943.

[9] A. Occhiello, F. Bentivegna, A. Borrelli, A. Schiattarella, A. Mancini and A. Pica, "Skin Necrosis in Sea Turtle Cold Stunning: Regeneration Following rMnSOD Topic Treatment in a Specimen of Caretta caretta," Comparative Clinical Pathology, Vol. 18, No. 4, 2009, pp. 365369. doi:10.1007/s00580-009-0816-9

[10] A. Mancini, A. Borrelli, M. T. Masucci, A. Schiattarella, S. Filice, J. Rashan and T. Maggino, “A Conditioned Medium from a Human Liposarcoma-Derived Cell Line Induces p53-Dependent Apoptosis in Several Tumor Cell Lines,” Oncology Reports, Vol. 7, No. 3, 2000, pp. 629637. 


\section{Abbreviations}

ROS: reactive oxygen species; UV: ultraviolet radiation; SODs: superoxide dismutases; MnSOD: manganese superoxide dismutase; rMnSOD: recombinant manganese superoxide dismutase. 